\title{
HIGH RATE OF COLISTIN AND FOSFOMYCIN RESISTANCE AMONG CARBAPENEMASE- PRODUCING ENTEROBACTERIACEAE IN TURKEY
}

\author{
SERAP SÜZÜK YILDIZ ${ }^{1 *}$, BANU KAŞKATEPE ${ }^{2}$, HÜSNIYE ŞIMŞEK ${ }^{1}$ and \\ FATMA MUTLU SARIGÜZEL ${ }^{3}$ \\ ${ }^{1}$ Department of National AMR Surveillance Laboratory, Public Health Microbiology \\ Reference Laboratories, Ministry of Health, Ankara, Turkey \\ ${ }^{2}$ Faculty of Pharmacy, Department of Pharmaceutical Microbiology, \\ Ankara University, Ankara, Turkey \\ ${ }^{3}$ Department of Clinical Microbiology, Ankara Training and Research Hospital, \\ Ankara, Turkey
}

(Received: 6 August 2018; accepted: 5 September 2018)

\begin{abstract}
When the problem with carbapenem-resistant Enterobacteriaceae (CRE) increases, the older antimicrobial agents such as colistin and fosfomycin are used for the treatment of these infections. In this study, the broth microdilution method for colistin and the agar dilution method for fosfomycin were used for a total of 147 multidrug-resistant (MDR) or extensively drug-resistant (XDR) strains of CRE. The study included Klebsiella pneumoniae (91.16\%), Escherichia coli (7.48\%), Enterobacter cloacae (0.68\%), and Serratia marcescens $(0.68 \%)$. All these strains produce various types of carbapenemase, including OXA-48, NDM, and KPC. Some of these strains also have three different carbapenemase mechanisms, including OXA-48 (78.23\%), NDM (2.04\%), and KPC $(0.68 \%)$ or OXA-48 and NDM $(10.88 \%)$, or OXA-48 and KPC $(0.68 \%)$. About $76.19 \%$ of the strains and $67.35 \%$ of the strains were resistant for colistin and fosfomycin, respectively. A total of 21 out of 35 colistin-susceptible strains were found to be susceptible to fosfomycin. This study showed that the resistance rates of colistin and fosfomycin are high. The MDR and XDR strains of CRE are spreading in our region and thus a monitoring system for CRE should be followed. Moreover, the applicability of antimicrobial stewardship programs should be increased in all inpatient and outpatient settings.
\end{abstract}

Keywords: carbapenem-resistant Enterobacteriaceae, colistin, fosfomycin, broth microdilution, agar dilution

*Corresponding author; Email: serapsuzuk@gmail.com 


\section{Introduction}

The strains of carbapenem-resistant Enterobacteriaceae (CRE) are a serious threat to public health with a rapid spread worldwide. The carbapenemases identified in Enterobacteriaceae are Klebsiella pneumoniae carbapenemase (KPC), the metallo- $\beta$-lactamases such as New Delhi metallo- $\beta$-lactamase (NDM), Verona integron-encoded metallo- $\beta$-lactamase, and imipenemase as well as OXA-48. All these enzymes hydrolyze penicillins and imipenem, sparing the broad-spectrum cephalosporins [1]. According to the data of the Central Asian and Eastern European Surveillance of Antimicrobial Resistance (CAESAR), the carbapenem resistance rates of Escherichia coli and $K$. pneumoniae, isolated from blood culture in Turkey in the year 2016, were $5 \%$ and $35 \%$, respectively [2]. In Istanbul, Turkey, the Ambler class D $\beta$-lactamase OXA-48 was initially identified from a carbapenem-resistant isolate of $K$. pneumoniae. In our country, $\beta$-lactamase OXA-48 is the most common carbapenemase, but recently, the association between OXA-48 and NDM-1 has been reported frequently [3, 4].

An important feature of the CRE is their resistance to multiple antibiotics. As a result, the treatment options with antibiotics are limited for these multidrug-resistant infections. Newer treatment options with antibiotics are very limited in the multidrugresistant Gram-negative bacteria. The old antibiotics such as colistin and fosfomycin are frequently used in the treatment of these isolates worldwide [5]. Colistin (polymyxin E) is one of the oldest antibiotics that affect the outer cell membrane of the Gram-negative bacilli by binding to the lipid A component of the lipopolysaccharide layer. The antibacterial spectrum of colistin includes most members of the Enterobacteriaceae family. Another old antibiotic is fosfomycin that inhibits bacterial cell wall synthesis [6]. In Turkey, colistin is used for the treatment of the multidrugresistant strains of CRE by either monotherapy or combination therapy [7]. Although the intravenous formulation of fosfomycin (fosfomycin disodium) is not available in Turkey, its use in the treatment of CRE has been reported to be quite safe [8].

Given the increase in CRE and multidrug resistance, this study was aimed to evaluate the frequency of colistin and fosfomycin resistance in CRE by the reference methods.

\section{Materials and Methods}

Settings and study design

This was a prospective study that included consecutive inpatients of any age and sex, having an infection with any CRE in the period between January 2017 
and June 2018. During this period, a total of 147 strains of CRE, referred to outside reference laboratories, were isolated from blood, urine, and tracheal aspirate. Using standard microbiology laboratory techniques, the clinical samples were analyzed. Only one isolate per patient was included. All the bacterial isolates were identified by matrix-assisted laser desorption ionization time of flight/mass spectrometry (Bruker MALDI Biotyper; Bruker Daltonics; Bremen, Germany).

Phenotypic extended-spectrum beta-lactamase (ESBL), ampicillin (AmpC), and carbapenemase tests

Antibiotic susceptibility tests were conducted and the ESBLs from the isolates were analyzed by the BD Phoenix automated system (BD Diagnostics, Sparks, MD). Meropenem disks $(10 \mu \mathrm{g})$ were used to screen the carbapenem resistance in all the isolates. If the diameter of the disk zones was found to be $<28 \mathrm{~mm}$, the production of carbapenemase was investigated [9]. ESBL, $\mathrm{AmpC}$, and carbapenemase were tested using the phenotypical Mast D68C $\mathrm{AmpC}+\mathrm{ESBL}$ Detection Set and the Mast D70C Carbapenemase Detection Set (Mast Diagnostics, UK). The Mastdisks ID inhibitor combination disks (Mast Diagnostics) method was performed according to the manufacturer's instructions.

Detection of the genes for resistance to carbapenem

The $b l a_{\mathrm{KPC}}, b l a_{\mathrm{OXA}-48}$, and $b l a_{\mathrm{NDM}-1}$ genes were detected in the isolates by the polymerase chain reaction method, displaying a reduced sensitivity against meropenem [3, 10-13].

Determination of the minimum inhibitory concentration (MIC) value for colistin by the broth microdilution method

The MIC value for colistin was determined by the broth microdilution method, using the cation-adjusted Mueller-Hinton broth (Oxoid, Code: CM0405, UK), according to the ISO Standard 20776-1:2006 [14]. Colistin sulfate (Sigma-Aldrich, St. Louis, MO) was tested over a range of dilutions $(0.06-64 \mu \mathrm{g} / \mathrm{ml})$. An amount of $50 \mu \mathrm{l}$ of the different concentrations of freshly prepared antibiotics was added to 96-well U-bottom microplates. Bacterial suspensions, prepared from the bacteria, grown in non-selective culture media, were added to the microplates. The microplates were then incubated at $37^{\circ} \mathrm{C}$ for $24 \mathrm{~h}$ in ambient air. The breakpoints of the European Committee on 
Antimicrobial Susceptibility Testing (EUCAST) were used as references for comparing the results (susceptible $\leq 2 \mathrm{mg} / \mathrm{L}$; resistant $>2 \mathrm{mg} / \mathrm{L}$ ) [15].

Determination of the MIC value for fosfomycin by the agar dilution method

The agar dilution method was performed according to the guidelines of the Clinical and Laboratory Standards Institute [16]. Mueller-Hinton agar plates (Oxoid, UK) containing $25 \mathrm{mg} / \mathrm{L}$ glucose-6-phosphate and fosfomycin at concentrations ranging from 0.25 to $1.28 \mathrm{mg} / \mathrm{L}$ were prepared and then an inoculum of $10^{4} \mathrm{CFU} / \mathrm{ml}$ was introduced onto the agar plates by a multipoint inoculator and allowed to dry. The agar plates were then incubated at $35^{\circ} \mathrm{C}$ for $16-20 \mathrm{~h}$. After incubation, the MIC value was determined as the lowest concentration without any visible growth. The breakpoints of the EUCAST were used as the references for comparing the results (susceptible $\leq 32 \mathrm{mg} / \mathrm{L}$; resistant $>32 \mathrm{mg} / \mathrm{L}$ ) [17].

$E$. coli ATCC 25922 and E. coli NCTC 13846 were used as quality control strains for the broth microdilution and agar dilution methods.

\section{Statistical analysis}

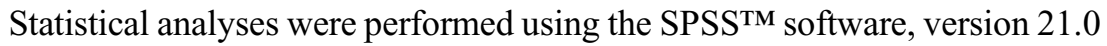
(IBM Corp., New York, NY). The results are presented as descriptive statistics and expressed in terms of relative frequency.

\section{Results}

A total of 147 CRE strains were evaluated in this study, which included 134 strains of $K$. pneumoniae $(91.16 \%), 11$ strains of E. coli $(7.48 \%), 1$ strain of Enterobacter cloacae (0.68\%), and 1 strain of Serratia marcescens $(0.68 \%)$. The ESBL and AmpC positivity rates of the CRE strains were $20.41 \%$ and $5.44 \%$, respectively. Only in one strain of $E$. coli, AmpC with loss of porin was observed.

The strains were positive for the carbapenemases, such as OXA-48, NDM-1, and KPC. In addition, some of the isolates were positive for OXA-48 and NDM or OXA-48 and KPC together. The positivity distributions are shown in Table I.

The rates of resistance to colistin and fosfomycin were found to be $76.19 \%$ and $67.35 \%$, respectively. A total of 87 out of 112 colistin-resistant strains $(59.18 \%)$ were found to be resistant to fosfomycin, whereas a total of 21 out of 35 colistin-susceptible strains $(60 \%)$ were found to be susceptible to fosfomycin. Furthermore, a total of 20 out of 30 isolates positive for both ESBL and 


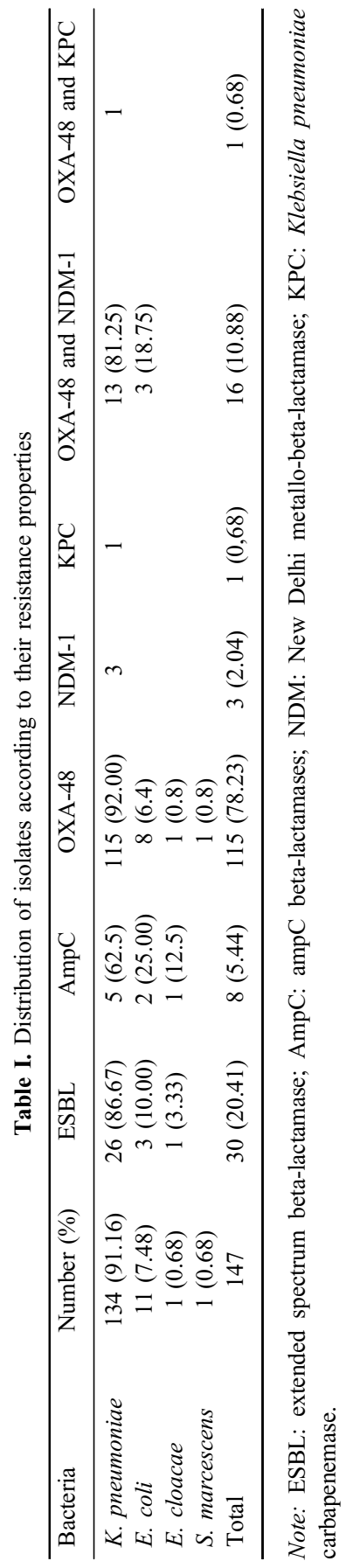


carbapenemase were found to be resistant to colistin and fosfomycin. Five AmpC positive strains were resistant to both colistin and fosfomycin.

Susceptibility to colistin (MIC 50 and $M I C_{90}$ values)

A total of 112 out of 147 strains $(76.19 \%)$ were found to be resistant to colistin. The MIC values for colistin were determined by the broth microdilution technique on all the strains. The $\mathrm{MIC}_{50}$ and $\mathrm{MIC}_{90}$ values for colistin were found to be 32 and $64 \mathrm{mg} / \mathrm{L}$, respectively.

Susceptibility to fosfomycin (MIC $C_{50}$ and $M I C_{90}$ values)

A total of 99 out of 147 strains $(67.35 \%)$ were found to be resistant to fosfomycin. The agar dilution method was used to determine fosfomycin susceptibility for all the strains. The $\mathrm{MIC}_{50}$ and $\mathrm{MIC}_{90}$ values for fosfomycin were found to be 64 and $128 \mathrm{mg} / \mathrm{L}$, respectively.

\section{Discussion}

The increase in the multidrug-resistant (MDR) and extensively drugresistant (XDR) strains of bacteria is a global issue. The limited availability of new antibiotic options, the inadequate implementation of control measures for infections, and the irrational usage of antibiotic policies further contribute to the growth of this problem. The CAESAR data show that Turkey has a very high number of resistant isolates, and a high percentage of these strains also have multidrug resistance [2].

Despite their potential side effects, colistin and fosfomycin are known to provide a therapeutic option for the MDR or XDR strains of Enterobacteriaceae. This study showed very high resistance rates of $76.19 \%$ and $67.35 \%$ to colistin and fosfomycin, respectively, among the MDR and XDR strains of Enterobacteriaceae. The reason behind such high rates of resistance is that only the XDR and MDR isolates were selected for this study. Moreover, a study on antibiotic consumption among patients, hospitalized in Turkey, showed that $44.8 \%$ of them were using antibiotics and the total antibiotic consumption was 674.5 defined daily dose/1000 patient-days [18]. Therefore, it is highly possible that a high rate of resistance is associated with antibiotic consumption.

Although resistance to colistin by chromosomal mutations has been reported earlier, the plasmid-mediated colistin resistance gene, $m c r-1$, and its variants and 
two other genes, $m c r-1.2$ and $m c r-2$, have been reported in recent years [19, 20]. Although the presence of the plasmid-mediated colistin resistance genes, $m c r-1$ and $m c r-2$, is not detected in Turkey, the resistance to colistin, a last-line antibiotic against the infections of multidrug-resistant or carbapenem-resistant Gramnegative bacteria, is too high [21].

The development of resistance to fosfomycin is similar to that of colistin. As reported earlier, resistance to fosfomycin mainly occurs through chromosomal mutations. Subsequently, the plasmid-mediated mechanisms of resistance to fosfomycin have been described in the clinical strains of Gram-negative bacteria [22]. An increase in the plasmid-mediated mechanisms of resistance further increases the magnitude of this global problem of antibiotic resistance and thus it is necessary to give importance to the studies that need to be carried out for the monitoring and controlling of antibiotic resistance.

In a study conducted in a tertiary hospital in Pakistan, the rates of resistance to colistin and fosfomycin among the strains of CRE were found to be $15.9 \%$ and $12.3 \%$, respectively. In this study, the colistin susceptibility test was performed by the broth microdilution technique and the fosfomycin susceptibility test was performed by the disk diffusion method, using fosfomycin trometamol disks [23]. EUCAST does not suggest using fosfomycin trometamol disks for the strains of Enterobacteriaceae except for the urinary E. coli strains [9]. Fosfomycin has been shown to be slightly less active, especially against the KPC-producing strains of K. pneumoniae [24]. In this study, two of the KPC-producing strains were found to be resistant to fosfomycin.

In a study conducted in Germany in 2013, the susceptibility to fosfomycin, using the agar dilution method was determined to be $78 \%$ with the MIC50 and MIC90 values of 8 and $512 \mathrm{mg} / \mathrm{L}$, respectively [25]. Such a difference in the rates of resistance can be attributed to the MDR and XDR strains included in this study and also to the rates of resistance in Turkey, which are higher than in Germany. The MIC90 value for fosfomycin was found to be $128 \mathrm{mg} / \mathrm{L}$, but it might be more because the higher dilutions were not studied.

The carbapenemase, OXA-48, known to hydrolyze penicillins and carbapenems possesses poor activity against the broad-spectrum cephalosporins. Multidrug resistance in the OXA-48-producing strains often results from the co-production of the various mechanisms of resistance, particularly the ESBLs and other determinants of resistance [26]. In this study, the strains with ESBL or AmpC positivity were found to be resistant to both colistin and fosfomycin, whereas the rates of resistance to ESBL and AmpC were not found to be high.

We know that this study has a few limitations. We could not detect the genes for resistance to colistin and fosfomycin due to financial limitations. The other limitation of this study was that only the MDR and XDR strains were included in 
the study and thus the resistance rate was found to be too high. The fact that higher dilutions were not studied for the determination of MIC values could be another important limitation of this study.

\section{Conclusions}

However, this study is the first report on the susceptibility to colistin and fosfomycin, as determined by the reference methods from Turkey. According to the data obtained from this study, the use of colistin and fosfomycin does not seem to be a promising therapeutic approach for the MDR and XDR strains of Enterobacteriaceae in Turkey, and a regular monitoring system for the MDR and XDR strains of CRE is needed. Moreover, in order to identify the susceptible isolates, it should be ensured that the reference methods are applied in the laboratory. If necessary, support should be taken from the reference or national laboratories for the agar dilution and broth microdilution techniques.

\section{Conflict of Interest}

The authors declare no conflict of interest.

\section{References}

1. Tzouvelekis, L. S., Markogiannakis, A., Psichogiou, M., Tassios, P. T., Daikos, G. L.: Carbapenemases in Klebsiella pneumoniae and other Enterobacteriaceae: An evolving crisis of global dimensions. Clin Microbiol Rev 25, 682-707 (2012).

2. WHO Regional Office for Europe: Central Asian and Eastern European Surveillance of Antimicrobial Resistance Annual Report 2016. WHO Regional Office for Europe, Copenhagen, Denmark, 2016.

3. Poirel, L., Heritier, C., Tolün, V., Nordmann, P.: Emergence of oxacillinase-mediated resistance to imipenem in Klebsiella pneumoniae. Antimicrob Agents Chemother 48, 15-22 (2004).

4. Yıldız, S. S., Kaşkatepe, B., Avcıküçük, H., Öztürk, Ş.: Performance of CarbaNP and CIM tests in OXA-48 carbapenemase-producing Enterobacteriaceae. Acta Microbiol Immunol Hung 64, 9-16 (2017).

5. Falagas, M. E., Grammatikos, A. P., Michalopoulos, A.: Potential of old generation antibiotics to address current need for new antibiotics. Expert Rev Anti Infect Ther 6, 593-600 (2008).

6. Yamamoto, M., Pop-Vicas, A. E.: Treatment for infections with carbapenem-resistant Enterobacteriaceae: What options do we still have? Crit Care 18, 229-37 (2014).

7. Palacios-Baena, Z. R., Gutiérrez-Gutiérrez, B., Calbo, E., Almirante, B., Viale, P., Oliver, A., Pintado, V., Gasch, O., Martínez-Martínez, L., Pitout, J., Akova, M., Peña, C., 
Molina Gil-Bermejo, J., Hernández, A., Venditti, M., Prim, N., Bou, G., Tacconelli, E., Tumbarello, M., Hamprecht, A., Giamarellou, H., Almela, M., Pérez, F., Schwaber, M. J., Bermejo, J., Lowman, W., Hsueh, P. R., Paño-Pardo, J. R., Torre-Cisneros, J., Souli, M., Bonomo, R. A., Carmeli, Y., Paterson, D. L., Pascual, Á., Rodríguez-Baño, J., Spanish Network for Research in Infectious Diseases (REIPI)/European Study Group of Bloodstream Infections and Sepsis (ESGBIS)/INCREMENT Group: Empiric therapy with carbapenem-sparing regimens for bloodstream infections due to extended-spectrum $\beta$-lactamase-producing Enterobacteriaceae: Results from the INCREMENT cohort. Clin Infect Dis 65, 1615-1623 (2017).

8. Livermore, D. M., Warner, M., Mushtaq, S., Doumith, M., Zhang, J., Woodford, N.: What remains against carbapenem-resistant Enterobacteriaceae? Evaluation of chloramphenicol, ciprofloxacin, colistin, fosfomycin, minocycline, nitrofurantoin, temocillin and tigecycline. Int J Antimicrob Agents 37, 415-419 (2011).

9. EUCAST: EUCAST Guidelines for Detection of Resistance Mechanisms and Specific Resistances of Clinical and/or Epidemiological Importance Version 2.0. Available at http://www.eucast.org/fileadmin/src/media/PDFs/EUCAST_files/Resistance_mechanisms/ EUCAST_detection_of_resistance_mechanisms_v2.0_20171211

10. Schechner, V., Straus-Robinson, K., Schwartz, D., Pfeffer, I., Tarabeia, J., Moskovich, R., Chmelnitsky, I., Schwaber, M. J., Carmeli, Y., Navon-Venezia, S.: Evaluation of PCR-based testing for surveillance of KPC-producing carbapenem resistant members of the Enterobacteriaceae family. J Clin Microbiol 47, 3261-3265 (2009).

11. Mendes, R. E., Kiyota, K. A., Monteiro, J., Castanheira, M., Andrade, S. S., Gales, A. C., Pignatari, A. C., Tufik, S.: Rapid detection and identification of metallo-beta-lactamase encoding genes by multiplex real-time PCR assay and melt curve analysis. J Clin Microbiol 45, 544-547 (2007).

12. Biendo, M., Canarelli, B., Thomas, D., Rousseau, F., Hamdad, F., Adjide, C., Laurans, G., $\mathrm{Eb}, \mathrm{F}$. : Successive emergence of extended-spectrum beta-lactamase producing and carbapenemase-producing Enterobacter aerogenes isolates in a university hospital. J Clin Microbiol 46, 1037-1044 (2008).

13. Poirel, L., Dortet, L., Bernabeu, S., Nordmann, P.: Genetic features of bla $a_{\mathrm{NDM}-1}$-positive Enterobacteriaceae. Antimicrob Agents Chemother 55, 5403-5407 (2011).

14. ISO 20776-1:2006: Clinical laboratory testing and in vitro diagnostic test systems Susceptibility testing of infectious agents and evaluation of performance of antimicrobial susceptibility test devices - Part 1: Reference method for testing the in vitro activity of antimicrobial agents against rapidly growing aerobic bacteria involved in infectious diseases. ISO, Geneva, Switzerland, 2006.

15. European Committee on Antimicrobial Susceptibility Testing: Breakpoint tables for interpretation of MICs and zone diameters Version 8.1, valid from 2018-05-15. Available at http://www.eucast.org/fileadmin/src/media/PDFs/EUCAST_files/Breakpoint_tables/v_8.1_ Breakpoint_Tables.pdf

16. Clinical and Laboratory Standards Institute: Methods for dilution antimicrobial susceptibility tests for bacteria that grow aerobically; approved standard (8th ed.). CLSI document M7-A8. Clinical and Laboratory Standards Institute, Wayne, PA, 2009.

17. European Committee for Antimicrobial Susceptibility Testing (EUCAST) of the European Society of Clinical Microbiology and Infectious Dieases (ESCMID): EUCAST Definitive Document E.DEF 3.1, June 2000: Determination of minimum inhibitory 
concentrations (MICs) of antibacterial agents by agar dilution. Clin Microbiol Infect 6, 509-515 (2000).

18. Guclu, E., Ogutlu, A., Karabay, O., Demirdal, T., Erayman, I., Hosoglu, S., Turhan, V., Erol, S., Oztoprak, N., Batirel, A., Altay, F. A., Kaya, G., Karahocagil, M., Sozen, H., Yildirim, M., Kocak, F., Teker, B.: Antibiotic consumption in Turkish hospitals; a multicentre point prevalence study. J Chemother 29, 19-24 (2017).

19. Di Pilato, V., Arena, F., Tascini, C., Cannatelli, A., Henrici De Angelis, L., Fortunato, S., Giani, T., Menichetti, F., Rossolini, G. M.: mcr-1.2, a new mor variant carried on a transferable plasmid from a colistin-resistant KPC carbapenemase producing Klebsiella pneumoniae strain of sequence type 512. Antimicrob Agents Chemother 60, 5612-5615 (2016).

20. Xavier, B. B., Lammens, C., Ruhal, R., Kumar-Singh, S., Butaye, P., Goossens, H., Malhotra-Kumar, S.: Identification of a novel plasmid mediated colistin-resistance gene, mcr-2, in Escherichia coli, Belgium, June 2016. Euro Surveill 21, 1-6 (2016).

21. Sarı, A. N., Süzük, S., Karatuna, O., Öğünç, D., Karakoç, A. E., Çizmeci, Z., Alışkan, H. E., Cömert, F., Bakıı, M. Z., Akpolat, N., Çilli, F. F., Zer, Y., Karataş, A., Akgün Karapınar, B., Bayramoğlu, G., Özdamar, M., Kalem, F., Delialioğlu, N., Aktaş, E., Yılmaz, N., Gürcan, Ş., Gülay, Z.: Results of a multicenter study investigating plasmid mediated colistin resistance genes ( $m c r-1$ and $m c r-2)$ in clinical Enterobacteriaceae isolates from Turkey. Mikrobiyol Bull 51, 299-303 (2017).

22. Karageorgopoulos, D. E., Wang, R., Yu, X. H., Falagas, M. E.: Fosfomycin: Evaluation of the published evidence on the emergence of antimicrobial resistance in Gram-negative pathogens. J Antimicrob Chemother 67, 255-268 (2012).

23. Qamar, S., Shaheen, N., Shakoor, S., Farooqi, J., Jabeen, K., Hasan, R.: Frequency of colistin and fosfomycin resistance in carbapenem-resistant Enterobacteriaceae from a tertiary care hospital in Karachi. Infect Drug Resist 10, 231-236 (2017).

24. Mezzatesta, M. L., La Rosa, G., Maugeri, G., Zingali, T., Caio, C., Novelli, A., Stefani, S.: In vitro activity of fosfomycin trometamol and other oral antibiotics against multidrugresistant uropathogens. Int J Antimicrob Agents 49, 763-766 (2017).

25. Kaase, M., Szabados, F., Anders, A., Gatermann, S. G.: Fosfomycin susceptibility in carbapenem-resistant Enterobacteriaceae from Germany. J Clin Microbiol 52, 1893-1897 (2014).

26. Pfeifer, Y., Schlatterer, K., Engelmann, E., Schiller, R. A., Frangenberg, H. R., Stiewe, D., Holfelder, M., Witte, W., Nordmann, P., Poirel, L.: Emergence of OXA-48-type carbapenemase-producing Enterobacteriaceae in German hospitals. Antimicrob Agents Chemother 56, 2125-2128 (2012). 